\title{
NOVASAR-S AND MARITIME SURVEILLANCE
}

\author{
Pasquale Iervolino, Raffaella Guida \\ Surrey Space Centre - University of Surrey \\ Guildford, UK \\ \{P.Iervolino, R.Guida\}@surrey.ac.uk
}

\begin{abstract}
The paper shows a new algorithm for ship-detection from Synthetic Aperture Radar (SAR) images. The algorithm consists of three main stages: pre-processing, detection and discrimination. In the pre-processing a land mask is obtained considering the different statistics between the sea and the land's backscattered field; the detection stage isolates the bright points over the sea background employing a Constant False Alarm (CFAR) method; while the ships are retrieved, in the discrimination step, by evaluating the scattering contributions of the possible targets detected in the previous stage. The algorithm is tested on an airborne $S$ band SAR image of Portsmouth harbor, similar to those that will become available with the upcoming UK SAR mission NovaSAR-S.
\end{abstract}

Index Terms - Synthetic Aperture Radar (SAR), NovaSAR-S, ship-detection, CFAR, scattering models.

\section{INTRODUCTION}

Maritime surveillance is a topic of utmost importance and growing interest since the authorities need to protect the marine environment, to control immigration and make borders safer, to fight against pirates and traffickers of all kinds and to guarantee the safety of navigation and the security in general. Furthermore the maritime surveillance represents a powerful means against the smuggling and is helpful in preventing the illegal immigration [1]. There are several ways to monitor these activities such as ground coastal (AIS - Automatic Identification System) and airborne sensors. Additionally, a useful support can be brought also by spaceborne sensors since they present better time coverage than airborne ones and better spatial coverage than traditional AIS system, allowing wide control in open sea areas. Moreover, spaceborne sensors are able to acquire images independently from national borders. For all these reasons they are likely to be used better and more to integrate traditional techniques to monitor ocean and marine areas. For a continuing monitoring, independent of daylight or weather conditions, Synthetic Aperture Radar (SAR) sensors on spaceborne platforms are the best option even if they are usually characterized by high mission costs. A
Philip Whittaker

\author{
SSTL Ltd, Guildford, UK
}

P.Whittaker@sstl.co.uk

novelty in this respect will be brought by the UK mission NovaSAR-S, developed by SSTL Ltd. and Astrium U.K. NovaSAR-S will be the first spaceborne small satellite SAR mission designed and produced at a cost comparable with that of optical missions.

The paper is organized as follows: in section 2 the main applications of the future NovaSAR-S sensors are introduced with a focus on maritime surveillance; in section 3 the new ship-detection algorithm is presented and all its steps are described in detail; in section 4 the preliminary outcomes are reported and, finally, future perspectives are briefly commented.

\section{NOVASAR-S AND MARITIME MODE}

NovaSAR-S, mission developed by SSTL Ltd. and Astrium U.K., will be a constellation of three or more SAR sensors. Each sensor will operate in S-band, a frequency still unexplored and, above all, from a low cost and lightweight satellite thanks to the highly efficient S-band solid state technology. The first of the three sensors will be launched in early 2015. Three are the main target applications of NovaSAR-S: disasters and flooding monitoring, forestry assessments (deforestation monitoring and prevention) and maritime surveillance (ship detection and tracking, oil spill detection and monitoring, iceberg and ice edge monitoring and tracking). The sensor will support flexible modes of operations, among which there is the Maritime Surveillance actually suitable for ocean and marine application thanks to really large swath $(750 \mathrm{Km})$ and short revisit-time (less than 1 day with one satellite and less than 8 hours considering 3 satellites). The main parameters for all the operational modes are summarized in Table I [2]; while in Table II, NovaSAR-S specifications, including orbital and radar parameters, are shown [2]. In the next section the methodology for a new ship-detection algorithm is presented.

\section{SHIP DETECTION SCHEME}

Ship detection is one of the main SAR applications since the ships are usually built from large metal sheet and therefore detectable in SAR imagery; commonly a ship detection algorithm consists of three stages: pre-processing (land 
TABLES I: NOVASAR-S BASELINE OPERATING MODES

\begin{tabular}{|c|c|c|c|c|c|c|c|}
\hline Mode & $\begin{array}{l}\text { Incidence } \\
\text { angle }\end{array}$ & $\begin{array}{c}\text { Spatial } \\
\text { Resolution }\end{array}$ & Swath & 1 satellite & 3 satellites & 1 satellite & 3 satellites \\
\hline ScanSAR & $16-29^{0}$ & $20 \mathrm{~m}$ & $50-100 \mathrm{~km}$ & 4.4 & 1.5 & 1.3 & 0.4 \\
\hline Maritime Surveillance & $48-75^{0}$ & $30 \mathrm{~m}$ & $750 \mathrm{~km}$ & 0.9 & 0.3 & 0.5 & 0.2 \\
\hline Stripmap & $16-31^{0}$ & $6 \mathrm{~m}$ & $13-20 \mathrm{~km}$ & 3.2 & 1.1 & 0.9 & 0.3 \\
\hline
\end{tabular}

TABLE II: NOVASAR-S SPECIFICATIONS

\begin{tabular}{|c|l|}
\hline $\begin{array}{c}\text { Imaging frequency } \\
\text { band }\end{array}$ & S-band (3.1-3.3 GHz) \\
\hline Antenna & $\begin{array}{l}\text { Microstrip patch phased } \\
\text { array (3m x 1m) }\end{array}$ \\
\hline Polaritazitions & HH, HV, VH, VV \\
\hline Design life & 7 years \\
\hline Mass & $\sim 400 \mathrm{~kg}$ \\
\hline Optimum orbit & $580 \mathrm{~km}$ \\
\hline Payload duty cycle & $>3 \mathrm{~min}$ per orbit \\
\hline Payload data memory & $544 \mathrm{GBytes}$ \\
\hline Downlink rate & $500 \mathrm{Mbps}$ \\
\hline
\end{tabular}

masking), detection (also known as prescreening) and discrimination [3]. The flow-chart of the ship detection scheme is shown in fig.1, where all the steps are highlighted and elements of novelty are introduced in each stage.

\subsection{Pre-processing}

First of all the discrimination between the land and the sea surface is needed. At this aim some studies register the SAR image with existing geographic maps [3], others apply an active contour based segmenting methods [4]. In this paper, instead, a statistical segmentation is performed in order to obtain a land mask. Two threshold values are chosen to identify the land pixels. The low threshold is set by the mean intensity of several sea regions augmented by three times the relative standard deviation; while the high threshold is set to infinity; this choice minimize the chance to include sea pixels in the final mask. Since the backscattering from the land and from reflecting objects over the sea background (ships, vessels, forts, buoys) appear in both cases much brighter than the sea clutter in the SAR image, it is possible to keep the targets out of the mask by choosing a minimum population value: the cluster of pixels whose dimension is smaller than the minimum population, which value is set by the operator, are not regarded as land even if their grey level belongs to the threshold interval.

\subsection{Detection}

The most common pre-screening algorithms are based on
CFAR (Constant False Alarm Rate) detectors, where the sea clutter is modeled according to a suitable statistical distribution. A commonly used statistical model is the Gaussian distribution. This is often applicable since the central limit theorem states that the average of a large number of identically distributed random variables tends to have a Gaussian distribution; obviously the more independent scatterers are in a resolution cell the more adequate the Gaussian model is. With this model it is possible to obtain a closed form for CFAR ship-detection algorithms with a computational saving compared to others methods adopting different distributions [5]. But the spatial resolution is tremendously improved in modern high resolution SARs and, consequently, the number of independent scatterers in the resolution cell is decreasing. In this framework the Gaussian is no more the distribution which better models the sea clutter but it has been figured out that the $\mathrm{K}$ distribution and the generalized gamma distribution work much better [6]. Once the statistical model has been established a threshold value is computed in order to obtain a constant false alarm rate:

$$
P F A=1-\int_{-\infty}^{T} f(x) d x=\int_{T}^{\infty} f(x) d x
$$

where $P F A$ is the false-alarm probability, $f(x)$ is the sea clutter distribution and $T$ is the threshold value.

CFAR models do not take the ship itself into account and simply search for anomalies in the ocean clutter and sometimes this may result in a high false-alarm rate which has to be decreased in the final discrimination step.

\subsection{Discrimination}

The detection's aim is to pass all targets and reject only obviously non-target like occurring clutter and it may result in a high false-alarm rate. The discrimination stage, instead, can be regarded as a coarse-to-fine false alarm reduction process, at the end of which, ideally, only ship targets are highlighted. In literature several discrimination algorithms are already introduced: in [7] shapes and textures features are extracted, while in [8] the covariance descriptor and a quadratic discrimination have been applied.

The novel discrimination process introduced in this paper is made up of three steps. First of all, the likely targets are gathered in clusters and a threshold $T_{\mathrm{CL}}$ is chosen and applied. The clusters whose dimension is lower than the clustering threshold are eliminated (clustering phase). The 


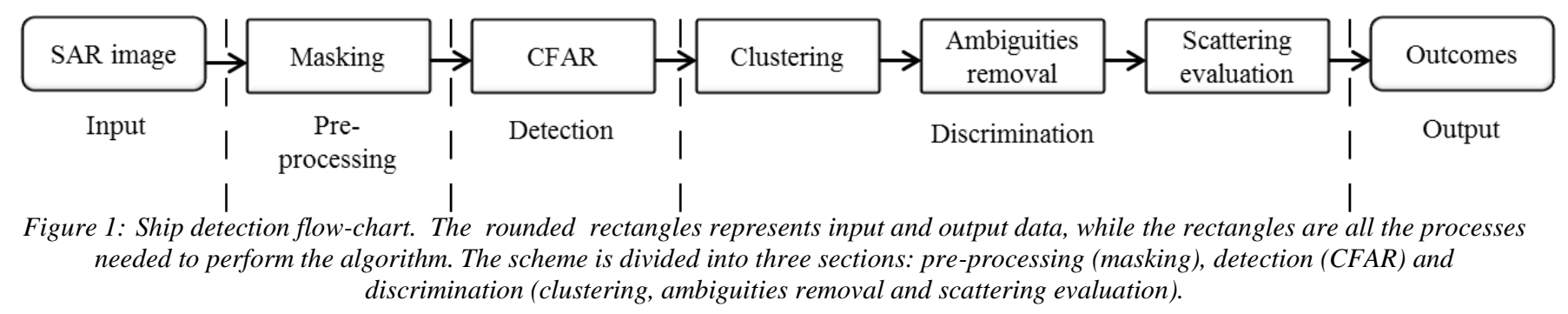

threshold has to be chosen according to the image resolution: the higher the resolution, the larger the threshold value has to be set. In the second step, the azimuth ambiguities, due to the side lobes of the azimuth antenna pattern, are removed by evaluating the relative distance between the target and the replica as performed in [5] (ambiguities removal). It is possible to evaluate the azimuth displacement between the target and the first order ambiguities considering the equation (2).

$$
\Delta x_{a z}=\frac{\lambda R f_{p}}{2 v}
$$

where $\Delta x_{a z}$ is the azimuth displacement, $R$ is the range distance between the target and the sensor, $\lambda$ is the wavelength, $f_{p}$ is the pulse repetition frequency and $v$ is the velocity of the sensor.

The last step (scattering evaluation) represents the main novelty introduced by this algorithm. It is possible to consider a simplified model of a canonical ship (complex target) by using a series of rectangular facets (parallelepiped representation). Since the different contributions (single scattering, layover, double and triple scattering) of the backscattered field from a simple parallelepiped are well known in literature [9], it is possible to reject all the clusters non-parallelepiped like.

In this work only the geometric model of the backscattering field is considered; the authors are currently working on the mathematical form of the scattering by such structures over a non-Gaussian surface. In the next section the algorithm proposed is applied to the case study.

\section{CASE STUDY AND RESULTS}

The novel procedure is tested on a dataset acquired from the Astrium airborne demonstrator in the Solent area (the harbor between Portsmouth and the Isle of Wight). In fig. 2(a) the amplitude of the image acquired on the $16^{\text {th }}$ June 2010 is shown. The polarization is $\mathrm{HH}$, the work frequency $3.2 \mathrm{GHz}$ (S-band) and the mean look-angle $(\theta)$ is $60^{\circ}$. The original dataset is mooltilooked in order to get a resolution similar to that of NovaSAR-S in the Stripmap mode $(6.6 \mathrm{~m}$ in slantrange and $7.0 \mathrm{~m}$ in azimuth) even if the signal-to-noise ratio will be different. In fig. 2(b), instead, the result of the masking procedure is displayed: the white pixels represent the sea surface while the black ones are the land and they are consequently excluded in the following CFAR algorithm.

In the detection stage, a Gaussian model is employed for the sea-clutter since the image presents a coarse resolution; a $P F A$ of $10^{-5}$ is set and the threshold value $T$ is consequently computed. In this case, as in [5], the detector is:

$$
x_{U T}>\mu+\sigma T \leftrightarrow \text { DETECTED }
$$

where $\mu$ and $\sigma$ are the sea clutter mean and standard deviation, respectively, and $x_{U T}$ is the pixel under test. In the clustering process all the isolated points $\left(T_{\mathrm{CL}}=1\right)$ are eliminated, the outcomes are shown in fig. 2(c): the red rectangles are the detected ships whose AIS signal is available; the green rectangles represent ship-like (parallelepiped-like scattering) targets whose AIS signal is unavailable; the yellow rectangles are azimuth ambiguities of land and ship targets rejected after the computation of (2) ( $\lambda=0.093 \mathrm{~m}, R=5070 \mathrm{~m}, f_{p}=121 \mathrm{~Hz}$ and $\nu=68.5 \mathrm{~m} / \mathrm{s}$ ); the red circles are the non parallelepiped-like clusters eliminated in the final scattering evaluation step.

In figs. 3-4, where the illumination comes from the left side, the different scattering mechanisms are shown for a non ship target (No Man's Land Fort, a cylindrical building located in the Solent area) and for a ship target (Celtic Fortune cargo). As expected from [10], in fig. 3(a) the two edge contributions of the cylinder are highlighted (points A and $\mathrm{B}$ ); the double-reflection and the layover (scattering from the roof and lateral wall) contributions are curve. In fig. 3(b) a constant azimuth cut is obtained and the different scattering contributions are located; moving from left to the right we find: scattering from the first edge (A), double reflection (second peak), layover area (third peak), scattering from the second edge (B). In addition, evaluating the distance between $\mathrm{A}$ and $\mathrm{B}$, it is possible to estimate the diameter of the fort [10]:

$$
\widehat{D}=A B / \sin \theta=69.5 \mathrm{~m}
$$

which is very close to the real dimension $(D=72 \mathrm{~m})$ and this enforces our study. Analogously, in fig. 4(a-b) the amplitude of the Celtic Fortune ship and the grey levels at a constant azimuth are reported. Differently from the cylinder, the parallelepiped-like does not show contributions from edges and the double reflection lays on a line, as written in [9].

All the AIS signals have been correctly detected, however the available ground truth is not complete and therefore it is not possible to carry out a full analysis of the performance. But it has been shown that the scattering evaluation is a good discriminator for targets of different shapes and the authors are currently working on the improvement of this particular aspect. 


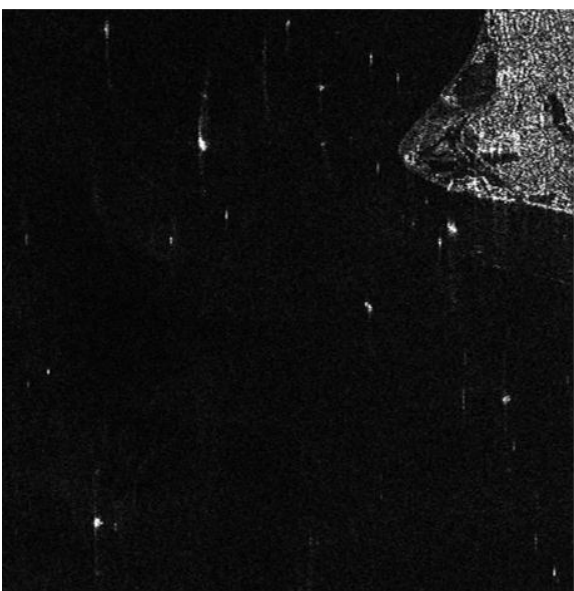

(a)

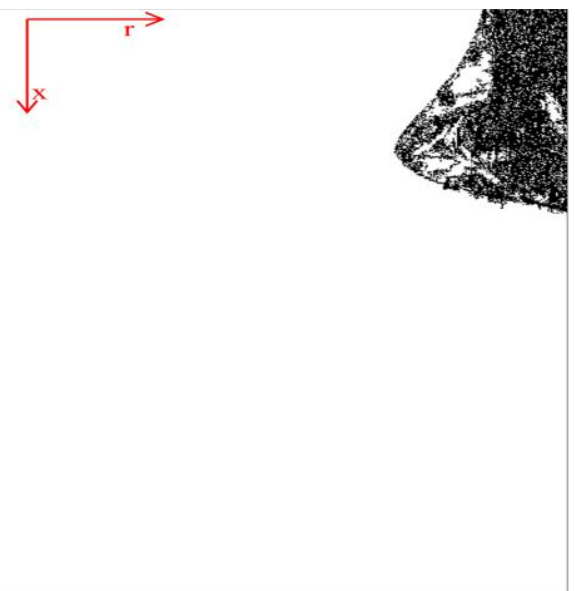

(b)

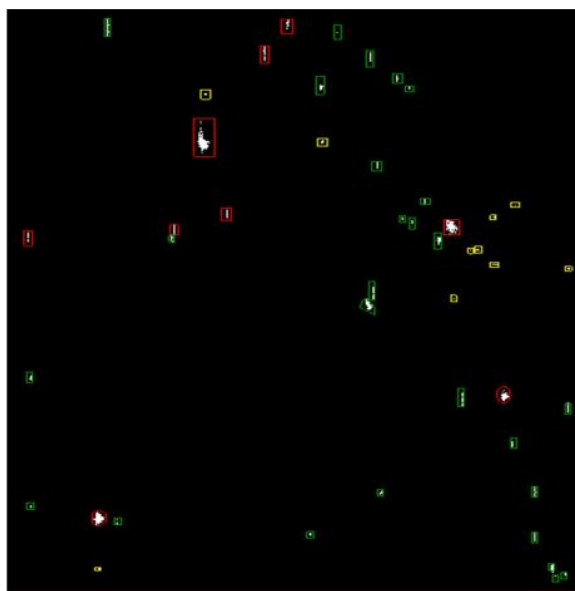

(c)

Figure 2: HH amplitude of the Solent harbor airborne image in slant range/azimuth projection (a). Results of the masking process (b). Outcomes (c):ships with available AIS data (red rectangles); ships or parallelepiped-like targets (green rectangles); azimuth ambiguities (yellow rectangles); non parallelepiped-like targets (red circles).

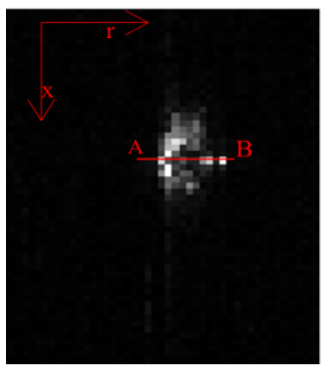

(a)

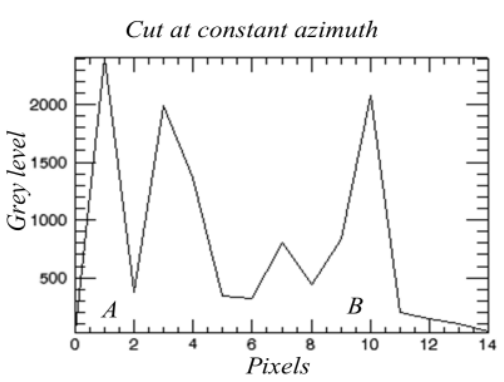

(b)
Figure 3:Amplitude of the No Man's Land Fort (a); cut at constant azimuth and relative grey levels $(b)$

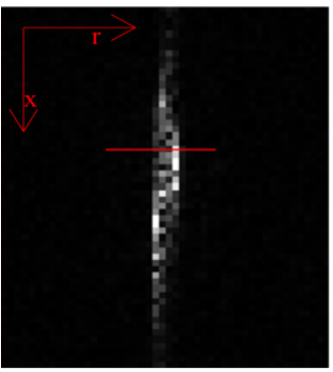

(a)

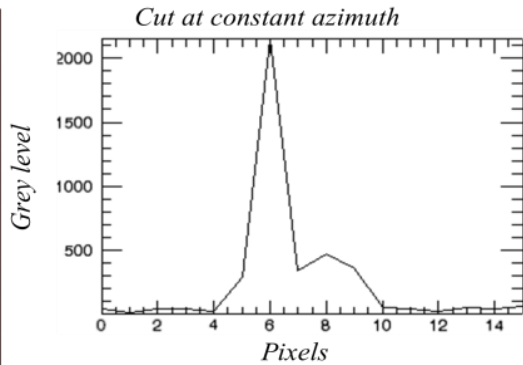

(b)
Figure 4:Amplitude of the Celtic Fortune ship (a); cut at constant azimuth and relative grey levels $(b)$

\section{REFERENCES}

[1] S. Ramongassie, N. Taveneau, T. Calmettes, J. Richard, R. Challmel, O. Autran, V. Foix, P. Durand, "Radar and AIS Sensors Constellation for Global Maritime Surveillance", Proceedings of the IEEE International Geoscience and Remote Sensing Symposium, pp. 3793-3796, Honolulu (Hawaii), 2010.

[2] P. Whittaker, M. Cohen, D. Hall, L. Gomes, "An Affordable Small Satellite SAR Mission", Proceedings of the 8th IAA
Symposium on Small Satellites for Earth Observation, Berlin (Germany), 2011.

[3] D. Crisp, "The State-of-Art in Ship Detection in SyntheticAperture Radar Imagery". Australia: DSTO Inf. Sci. Lab., 2004.

[4] Z. Shan, C. Wang, H. Zhang, B. Zhang, "Ocean-Land Segmentation Based on Active Contour Model for SAR Imagery". International Conference on Computer and Communication Technologies in Agriculture Engineering, pp. 215-218, 2010.

[5] S. Brusch, S. Lehner, T. Fritz, M. Soccorsi, A. Soloviev, B. van Schie, "Ship Surveillance With TerraSAR-X", IEEE Transactions on Geoscience and Remote Sensing, vol. 49, no 3, pp.1092-1103 March 2011.

[6] X. Qin, S. Zhou, H. Zou, G. Gao, "A CFAR Detection Algorithm for Generalized Gamma Distributed Background in High Resolution SAR Images, IEEE Geoscience and Remote Sensing Letters, vol. 10, no.10, pp. 806-810, July 2013.

[7] L. Novak, G. Owirka, C. Netishen, "Performance of a HighResolution Polarimetric SAR Automatic Target Recognition system", Lincolon Lab. J., vol. 6, no. 1, pp. 11-24, 1993.

[8] Y. Wang, H. Liu, "A Hierarchical Ship Detection Scheme for High-Resolution SAR Images", IEEE Transactions on Geoscience and Remote Sensing, vol. 50, no 10, pp.4173-4184 October 2012.

[9] G. Franceschetti, A. Iodice, D. Riccio, "A Canonical Problem in Electromagnetic Backscattering From Buildings", IEEE Transactions on Geoscience and Remote Sensing, vol. 40, no.8, pp. 1787-1801, August 2002.

[10] R. Guida, A. Iodice, D. Riccio, “Assessment of TerraSAR-X Products with a New Feature Extraction Application: Monitoring of Cylindrical Tanks", IEEE Transactions on Geoscience and Remote Sensing, vol. 48, no.2, pp. 930-938, February 2010. 